\title{
FNDC3B promotes epithelial-mesenchymal transition in tongue squamous cell carcinoma cells in a hypoxic microenvironment
}

\author{
ZHAOMING ZHONG ${ }^{1 *}$, HONGXING ZHANG ${ }^{1 *}$, MIN HONG $^{1}$, CHUANZHENG SUN $^{2}$, \\ YUANYUAN XU ${ }^{3}$, XIAO CHEN ${ }^{1}$, CHANGE GAO ${ }^{1}$, MINJIE HE ${ }^{1}$, WEIQING LIU ${ }^{1}$ and JIN LIANG ${ }^{1}$ \\ ${ }^{1}$ Department of Medical Oncology, The First Affiliated Hospital of Kunming Medical University; \\ ${ }^{2}$ Department of Head and Neck Surgery, The Third Affiliated Hospital of Kunming Medical University; \\ ${ }^{3}$ Department of Oncology, The First People's Hospital of Kunming, Kunming, Yunnan 650032, P.R. China
}

Received May 16, 2017; Accepted October 10, 2017

DOI: $10.3892 /$ or.2018.6231

\begin{abstract}
The primary reasons for the treatment failure of patients with oral tongue squamous cell carcinoma (OTSCC) are metastasis and tumor recurrence. Identifying the exact mechanisms underlying metastasis is a key point in improving patient prognosis. It has been reported that a hypoxic microenvironment plays an important role during the metastasis of malignancies. We found that the expression of fibronectin type III domain containing 3B (FNDC3B) is positively correlated with lymph node metastasis and advanced cTNM stage of OTSCC by IHC assay and correlation analysis. Furthermore, we found that knockdown of FNDC3B could suppress the migratory and invasive abilities of OTSCC cells. In addition, treating OTSCC cells with $\mathrm{CoCl}_{2}$ (a hypoxia mimetic agent) upregulated the mRNA and protein expression of FNDC3B via HIF-1 $\alpha$. Moreover, the resultant increase in FNDC3B expression significantly induced epithelial-mesenchymal transition (EMT) in OTSCC cells. The present study elucidated the important role played by FNDC3B in OTSCC metastasis and indicates FNDC3B as a potential target for the treatment of OTSCC metastasis. However, many questions remain to be explored.
\end{abstract}

\section{Introduction}

Head and neck squamous cell carcinoma (HNSCC) accounts for $90 \%$ of all head and neck cancers, and is the sixth most

Correspondence to: Professor Jin Liang, Department of Medical Oncology, The First Affiliated Hospital of Kunming Medical University, Kunming, Yunnan 650032, P.R. China

E-mail: liangjinkm@163.com

Professor Chuanzheng Sun, Department of Head and Neck Surgery, The Third Affiliated Hospital of Kunming Medical University, Kunming, Yunnan 650032, P.R. China

E-mail: scz007@126.com

*Contributed equally

Key words: tongue squamous cell carcinoma, FNDC3B, epithelialmesenchymal transition, metastasis, hypoxia-inducible factor $1 \alpha$ common malignancy worldwide (1). Despite improved locoregional control and more effective therapeutic strategies for HNSCC, the 5-year survival rate for HNSCC patients remains $50-60 \%$. Lymph node metastasis is one of the major reasons associated with the poor prognosis of patients with HNSCC (2). It is reported that the lymph node metastasis rate of oral tongue squamous cell carcinoma (OTSCC) is nearly $50 \%$, and lymph node metastasis is the primary factor influencing prognosis (3-5). Thus, understanding the mechanisms that underlie the metastasis of OTSCC is urgently needed to improve therapeutic strategies and ultimately patient prognosis.

In the present study, we investigated the mechanisms underlying the lymph node metastasis of OTSCC. FNDC3B (fibronectin type III domain containing 3B), also named FAD104, was found to be upregulated in tongue cancer through a web-based bioinformatic meta-analysis (Oncomine). FNDC3B is known to be a member of the FNDC3 family (including FNDC3A, FNDC3B and FNDC3C). FNDC3B has a proline-rich region, nine fibronectin type III domains and a transmembrane region (6,7). The fibronectin type III (FNIII) domains act as a scoffold and can integrate with different proteins, which plays an important role in cell adhesion and growth signaling (8). Initially, FNDC3B was identified as a known regulator of adipocyte and osteoblast differentiation, and FNDC3B-deficient mice died at birth due to lung abnormalities (9). Moreover, analyses using mouse embryonic fibroblasts (MEFs) revealed that loss of FNDC3B suppressed cell adhesion, migration and proliferation (10). These results suggest that FNDC3B has important roles in cell adhesion, migration and proliferation, which raised the question as to whether FNDC3B regulates the invasion and metastasis of cancer cells and promoted us to investigate its role in the metastasis of cancer cells. Previous studies have demonstrated that expression of FNDC3B protein was upregulated in a series of human tumors, including breast carcinoma (11), esophageal carcinoma (12), glioblastoma $(13,14)$ and hepatocellular carcinoma $(11,15)$. Moreover, FNDC3B has also been confirmed to serve as an oncogene and play a significant role in the regulation of the motility and invasion of glioma and hepatocellular carcinoma cells. However, there is little research concerning its biological functions, particularly in the metastasis of tongue squamous cell carcinoma. 
A hypoxic enviroment plays an important role in the metastasis of cancer cells (16). The induction of hypoxiainducible factor (HIF)-1 is the best-characterized transcription factor that is responsive to hypoxia (17). HIF-1 is composed of $\alpha \beta$ heterodimers; expression of the $\alpha$-subunit is tightly regulated by oxygen, whereas the $\beta$-subunit is constitutively expressed (18). Numerous studies have shown that HIF-1 $\alpha$ overexpression is frequently observed in human cancers and is associated with metastasis of several types of solid types of carcinoma, including breast carcinoma, chondrosarcoma, colorectal adenocarcinoma and head and neck cancer (19-21).

In the present study, we demonstrated that FNDC3B expression is positively correlated with lymph node metastasis and clinical tumor-node-metastasis (cTNM) stage in OTSCC patients. Furthermore, we demonstrated that cobalt chloride $\left(\mathrm{CoCl}_{2}\right.$, a hypoxia mimetic agent) promoted FNDC3B expression, and then induced EMT in OTSCC cells, ultimately promoting their migratory and invasive abilities. This study elucidated the important role played by FNDC3B in OTSCC metastasis and the identification of possible drug targets.

\section{Materials and methods}

Patients and clinical tissue specimens. The present study was approved by the Ethics Committee of the Kunming Medical University. All patients provided written informed consent in order to participate in the study. A total of 116 paraffin-embedded OTSCC samples, which were consecutively pathologically diagnosed between January 2004 and January 2014 at the First Affiliated Hospital of Kunming Medical University, were collected for immunohistochemical (IHC) analysis. These samples were obtained from 56 men and 60 women, with a mean age of 55 years (ranging from 35 to 86 years). All patients were followed up until July 2016. cTNM stages were assessed according to the TNM classification of the American Joint Committee on Cancer (AJCC) (22). Correlations between FNDC3B expression levels and clinical features in the patient cohort are shown in Table I.

Silico gene expression studies. We used the Oncomine database (Compendia Bioscience; http://www.oncomine.org) to identify upregulated genes in tongue carcinoma on April 23, 2017 and performed a microarray meta-analysis to compare all genes across 5 different datasets (23-27) that were identified by the following parameters: 'mRNA', 'tongue carcinoma' and 'cancer vs. normal analysis'. To compare FNDC3B expression between cancer and normal tissues, FNDC3B expression fold changes were limited to $\mathrm{P}<0.05$.

Immunohistochemical assay (IHC). Paraffin-embedded OTSCC tissue specimens were cut into $3-\mu \mathrm{m}$-thick sections and incubated at $60^{\circ} \mathrm{C}$ for $2 \mathrm{~h}$. All sections were deparaffinized with xylene and rehydrated with a gradient of ethanol to distilled water. After soaking with $3 \% \mathrm{H}_{2} \mathrm{O}_{2}$ for 15 min to block endogenous peroxidase, the sections were microwaved in sodium citrate buffer $(\mathrm{pH} \mathrm{8.0)}$ for antigen retrieval. Then, incubation was carried out with a rabbit anti-FNDC3B antibody (Proteintech Group, Rosemont, IL, USA) overnight at $4^{\circ} \mathrm{C}$ (dilution of 1:200). The slides were washed with phosphate-buffered saline (PBS) three times and incubated with anti-rabbit secondary antibody for $50 \mathrm{~min}$ at $37^{\circ} \mathrm{C}$. Diaminobenzidine (DAB; Zhongshan Biological and Technical Co., Beijing, China) was used as a colorimetric reagent for protein detection. Normal goat serum was used as a negative control. Semi-quantitative expression levels were measured by assessing the intensities and percentages of the stained cancer cells. The percentage of positive cancer cells was divided into four levels: 0 (0-25\%), 1 (6-50\%), 2 (51-75\%) and $3(76-100 \%)$. Staining intensity was divided into four grades: 0 (no staining), 1 (yellow), 2 (deep yellow) and 3 (brown). Scoring of both parameters was calculated after counting at least 4 fields. The overall score was the addition of the positive cell percentage and the staining intensity. Each specimen was classified into 1 of 2 groups according to overall scores: scores of $0-3$ points were classified as negative or low expression, and scores $>3$ points were classified as high expression for FNDC3B. All specimens were independently assessed by two pathologists who were blindly to the patient identity and clinical outcome.

Cell lines and cell culture. The tongue squamous cell carcinoma (TCA8113, TSCCA and CAL27) cell lines were purchased from the American Type Culture Collection (ATCC; Manassas, VA, USA). The human normal epithelial carcinoma cell line NTEC was a gift from Musheng Zeng (Sun Yat-sen University Cancer Center, Guangzhou, China). All cells were cultured in RPMI-1640 medium supplemented with $10 \%$ fetal bovine serum (FBS; Thermo Fisher Scientific, Inc., Waltham, MA, USA), streptomycin $(100 \mathrm{U} / \mathrm{ml})$ and penicillin $(100 \mathrm{U} / \mathrm{ml})$ in a humidified $5 \% \mathrm{CO}_{2}$ incubator at $37^{\circ} \mathrm{C}$.

Antibodies and reagents. Mouse anti-E-cadherin and antivimentin antibodies were obtained from BD Technologies (La Jolla, CA, USA). DMOG was obtained from Santa Cruz Biotechnology (Santa Cruz, CA, USA). Antibody against $\beta$-catenin was purchased from Cell Signaling Technology (Beverly, MA, USA). Rabbit anti-HIF-1 $\alpha$ antibody was purchased from Abcam (Cambridge, MA, USA), and mouse anti- $\beta$-actin antibody and anti-GAPDH were obtained from Proteintech Group (Chicago, IL, USA). Goat anti-rabbit and anti-mouse goat peroxidase-conjugated secondary antibodies were purchased from Pierce Biotechnology (Rockford, IL, USA). All other reagents were obtained from Sigma-Aldrich (St. Louis, MO, USA)

Generation of target-specific silenced cells. Retrovirus particles were produced in 293FT cells by transfection of pSuper-Retro-Puro plasmid harboring specific shRNA. The shRNAs were determined by Invitrogen's siRNA design tool (Invitrogen, Carlsbad, CA, USA). The target sequences were as follows: FNDC3B shRNA\#1: 5'-GCAGGTTATTCTCG TTCAA-3'; FNDC3B shRNA\#2: 5'-GCTTACTACCCACCT GTTA-3'; FNDC3B shRNA\#3: 5'-GCAGCTGCACAACA GTATA-3'. A standard calcium phosphate co-transfection was performed with a PIK packaging plasmid in the 293FT cells. The supernatant was collected and filtered using $0.45-\mu \mathrm{m}$ filters. The target cells were incubated with $2 \mu \mathrm{g} / \mathrm{ml}$ Polybrene (Sigma-Aldrich). Twenty-four hours after infection, the cells were incubated with $1 \mu \mathrm{g} / \mathrm{ml}$ puromycin for 3 days for selection of positive cells. 
Table I. Correlation between FNDC3B expression and clinicopathological features of the patients with OTSCC.

\begin{tabular}{lcccc}
\hline & \multicolumn{2}{c}{ FNDC3B } & & \\
\cline { 2 - 3 } Characteristics & Low & High & $\chi^{2}$ & P-value \\
\hline Sex & & & 0.534 & 0.577 \\
$\quad$ Male & 29 & 27 & & \\
$\quad$ Female & 27 & 33 & & \\
Age (years) & & & 0.011 & 1.000 \\
$\quad<50$ & 20 & 22 & & \\
$\geq 50$ & 36 & 38 & & \\
Clinical T phase & & & 4.388 & 0.045 \\
$\quad$ T1-2 & 51 & 46 & & \\
$\quad$ T3-4 & 5 & 14 & & \\
Clinical N phase & & & 5.712 & 0.022 \\
$\quad$ N0 & 41 & 31 & & \\
$\quad$ N1+2 & 15 & 29 & & \\
Histological differentiation & & & 0.267 & 0.709 \\
I & 30 & 35 & & \\
$\quad$ II-III & 26 & 25 & & \\
$\quad$ cTNM stage & & & 4.342 & 0.042 \\
I-II & 36 & 27 & & \\
III-IV & 20 & 33 & & \\
\hline
\end{tabular}

Western blotting. Cells were washed twice with PBS and then lysed in $1 \mathrm{X}$ sodium dodecyl sulfate (SDS) sample buffer. A total of $25 \mu \mathrm{g}$ protein was loaded on a 9\% SDS-polyacrylamide gel by electrophoresis, transferred to a polyvinylidene fluoride (PVDF) membrane at $100 \mathrm{~mA}$ for $2 \mathrm{~h}$, blocked with 5\% skim milk for $1 \mathrm{~h}$ in room temperature, and then incubated with the primary antibodies overnight at $4{ }^{\circ} \mathrm{C}$. On the folllowing day, incubation with the secondary antibodies was carried out at room temperature for $45 \mathrm{~min}$. After washing, bound antibodies were visualized via electrochemiluminescence, which was captured by XAR film. Scanning and analysis of the western bloting bands were performed using the Quantity One program (Bio-Rad Laboratories, Inc., Hercules, CA, USA).

Quantitative real-time polymerase chain reaction (real-time $P C R$ ). TRIzol reagent (Invitrogen) was used to extract total RNA from the cultured cells, and $2 \mu \mathrm{g}$ of each sample was reverse-transcribed using M-MLV reverse transcriptase (Promega, Madison, WI, USA) following the kit instructions. Real-time PCR was performed with the Fast SYBR-Green Master Mix (Bio-Rad Laboratories, Inc.). The housekeeping gene GAPDH was used as an internal control. The PCR amplifications were performed in a PTC-200 PCR system (Bio-Rad Laboratories, Inc.) using the following cycle parameters: $10 \mathrm{~min}$ at $95^{\circ} \mathrm{C}$, followed by 45 cycles of $10 \mathrm{sec}$ at $95^{\circ} \mathrm{C}, 10 \mathrm{sec}$ at $55^{\circ} \mathrm{C}$, and $20 \mathrm{sec}$ at $72^{\circ} \mathrm{C}$, with a final extension at $72^{\circ} \mathrm{C}$ for $10 \mathrm{~min}$. The FNDC3B sense primer was 5'-CCACCTGTTACCGGACCTG-3' and the antisense primer was 5'-GGGTGATGTAGGTTGACATTCC-3'. The mRNA relative expression levels were quantified using the $2^{-\Delta \Delta \mathrm{Ct}}$ method.

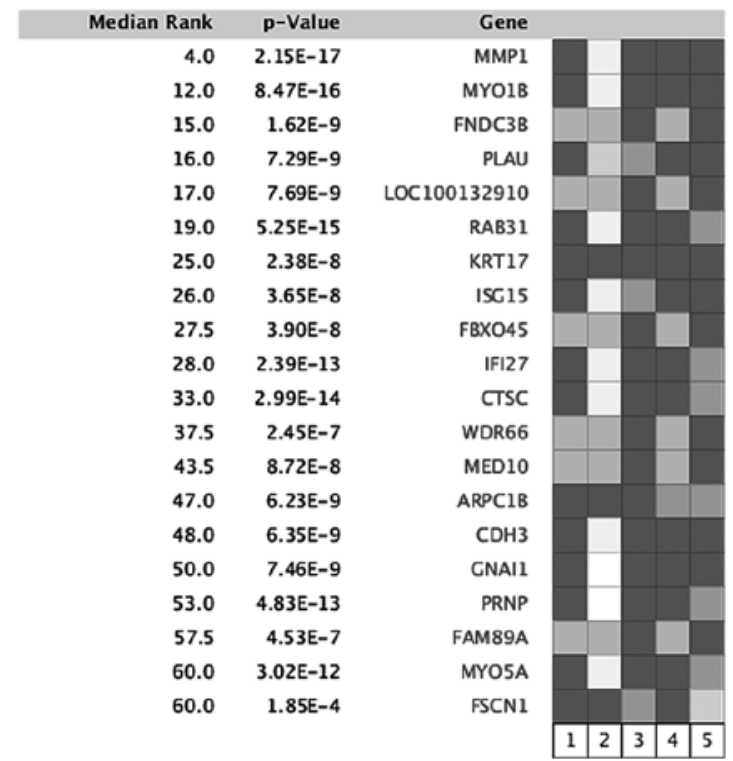

Figure 1. FNDC3B mRNA was found to be significantly upregulated in tongue carcinoma as shown in the Oncomine database. A microarray metaanalysis was performed using Oncomine to compare genes across 5 different datasets (Materials and methods) and FNDC3B was identified as one of the most frequently upregulated genes in tongue carcinoma tissues. The top 20 overexpressed genes identified by meta-analysis are shown

Cell invasion and migration assays. After being serum-starvation for $24 \mathrm{~h}, 4 \times 10^{4}$ cells were plated into a 24-well Transwell plate (Corning Costar, Cambridge, MA, USA) with or without a Matrigel coating (BD Biosciences, Bedford, MA, USA). A total of $500 \mu \mathrm{l}$ of RPMI-1640 supplemented with $10 \%$ FBS was placed in the lower chamber. After incubation at $37^{\circ} \mathrm{C}$ for 12 (migration) or $24 \mathrm{~h}$ (invasion), the cells that migrated to the reverse sides of the inserts were fixed in methanol and stained with $0.1 \%$ crystal violet (Sigma-Aldrich) for $10 \mathrm{~min}$, photographed and counted (6 random 100x fields per well) under an inverted microscope (Olympus Corp., Tokyo, Japan). Each experiment was repeated at least three times.

Statistical analysis. The SPSS statistical package 19.0 (SPSS, Inc., Chicago, IL, USA) was used to analyze the data. Data are presented as the means \pm SEM of values from three independent experiments. Correlations between FNDC3B expression and the clinical features of the OTSCC patient cohort were assessed by the $\chi^{2}$ test. The Student's t-test was used for multiple comparisons. $\mathrm{P}<0.05$ was considered statistically significant. Error bars represent SEM. ${ }^{*} \mathrm{P}<0.05,{ }^{* *} \mathrm{P}<0.01$, ${ }^{* * * *} \mathrm{P}<0.001$ were indicative of statistically significant results as shown in the figures. Each experiment was repeated at least three times.

\section{Results}

Overexpression of FNDC3B in OTSCC samples is related to the clinicopathological features of the OTSCC patients. To identify the molecules involved in lymph node metastasis of human OTSCC, a microarray meta-analysis was performed to compare all genes across 5 different datasets (including 107 cancer tissues and 87 adjacent normal tissues from OTSCC patients). The overexpressed genes were ranked by median 

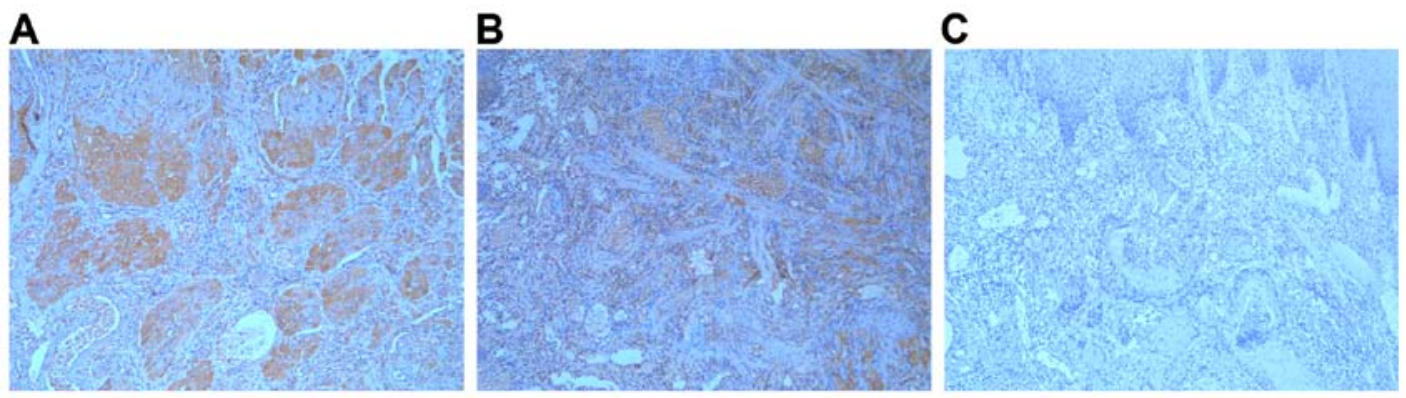

Figure 2. FNDC3B expression in paraffin-embedded OTSCC specimens. (A) High expression, (B) low expression, (C) negative expression of FNDC3B protein in OTSCC tissues. Original magnification, $\mathrm{x} 40$.

A

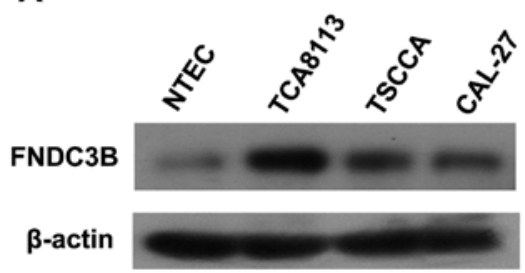

B

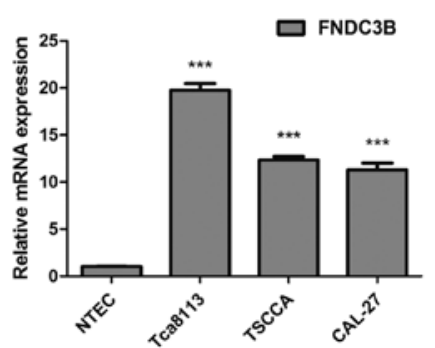

Figure 3. OTSCC cell lines exhibit increased FNDC3B expression. (A) Protein and (B) mRNA expression levels of FNDC3B were significantly higher in OTSCC cell lines (TCA8113, TSCCA and CAL27) than these levels noted in the normal tongue epithelial carcinoma cell line (NTEC), as measured by western blotting and real-time PCR, respectively. ${ }^{* * *} \mathrm{P}<0.001$.

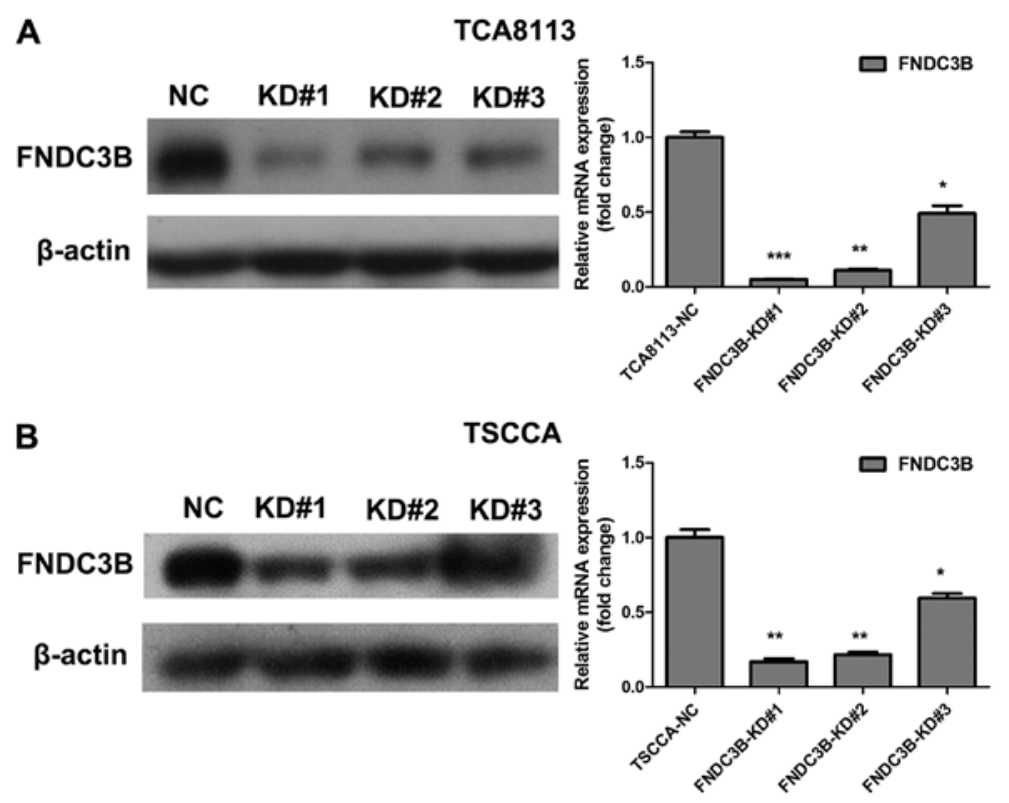

Figure 4. After knockdown of FNDC3B, the protein and mRNA levels of FNDC3B in (A) TCA8113 and (B) TSCCA cells were significantly decreased, as measured by western blotting and real-time PCR, respectively. ${ }^{*} \mathrm{P}<0.05,{ }^{* *} \mathrm{P}<0.01,{ }^{* * * *} \mathrm{P}<0.001$ compared with the NC-transfected cells.

ranked analyses, and FNDC3B was identified as one of the most frequently upregulated genes in OTSCC tissues compared to adjacent tissues (Fig. 1). Therefore, we further evaluated the roles of FNDC3B in OTSCC progression in the present study.

To assess the clinical significance of FNDC3B in OTSCC samples, we examined FNDC3B expression in 116 paraffinembedded OTSCC samples by immunohistochemistry. As a result, 56 cases $(48.3 \%)$ showed negative/low FNDC3B expression, and 60 (51.7\%) cases showed high FNDC3B expression (Fig. 2). In the correlation analysis, positive FNDC3B expression was associated with lymph node metastasis and advanced cTNM stage (Table I).

OTSCC cell lines exhibit increased FNDC3B expression. Real-time PCR and western blotting were conducted to compare FNDC3B expression in OTSCC cell lines (TCA8113, 
A

B NC KD\#1

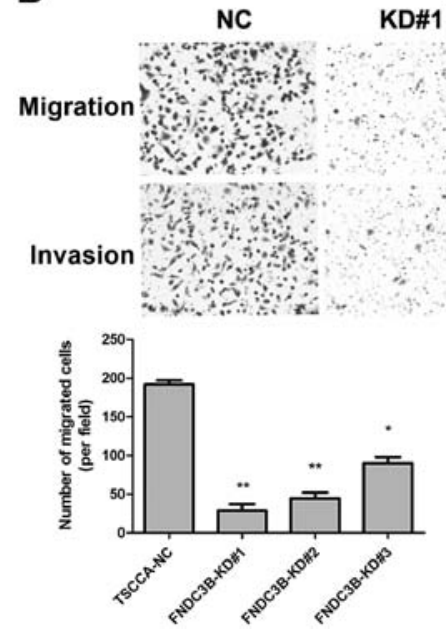

TSCCA

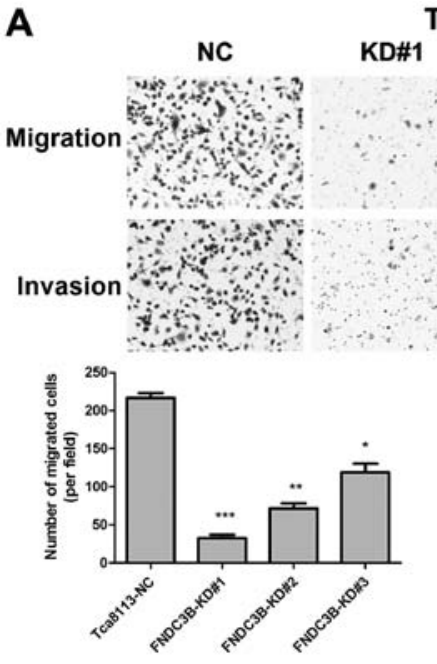

CA8113
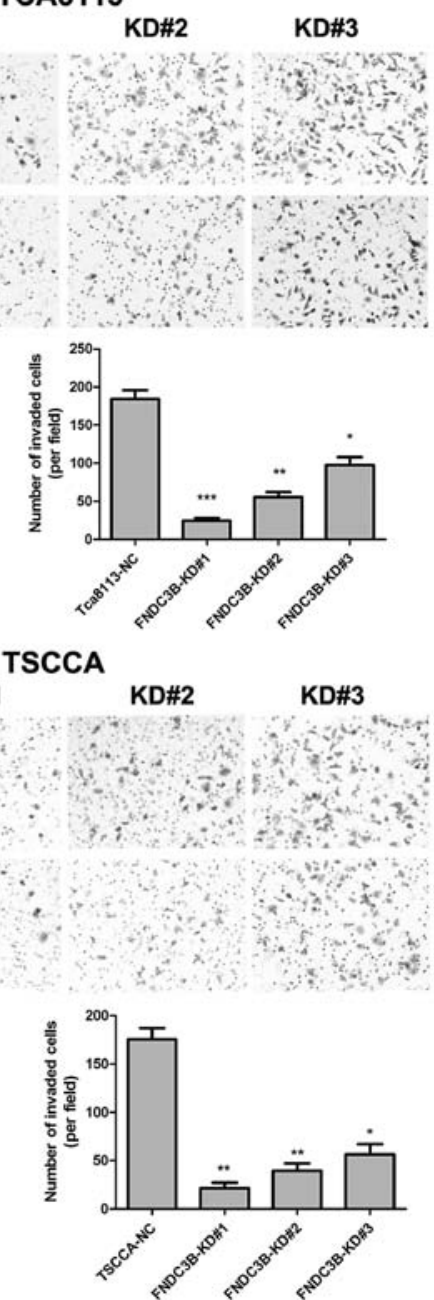

Figure 5. After knockdown of FNDC3B, the invasive and migratory abilities of (A) TCA8113 and (B) TSCCA cells were significantly decreased, as assessed by Transwell assay. ${ }^{*} \mathrm{P}<0.05,{ }^{* *} \mathrm{P}<0.01,{ }^{* * *} \mathrm{P}<0.001$ compared to the NC-transfected cells. Photomicrographs are at x100 magnification.

TSCCA and CAL27) and a normal tongue epithelial cell line (NTEC). Western blotting revealed higher FNDC3B protein expression in the OTSCC cell lines compared with the NTEC cells (Fig. 3A). Similar result was found for FNDC3B mRNA expression based on real-time PCR (Fig. 3B). Thus, our data indicated that FNDC3B mRNA and protein expression were significantly increased in the OTSCC cell lines.

FNDC $3 B$ is closely associated with the invasive and migratory abilities of OTSCC cells. To clarify the role of FNDC3B in OTSCC cell invasion and migration, we generated TCA8113 cells with stably knocked down FNDC3B expression using retroviral vectors (FNDC3B-KD\#1, KD\#2 and KD\#3), and scrambled shRNA was used as a negative control (NC). Real-time PCR and western blotting were performed to assess the FNDC3B knockdown efficiency. As shown in Fig. 4, FNDC3B mRNA and protein levels were significantly lower in FNDC3B-KD cells than NC cells, confirming the knockdown efficiency of FNDC3B in these cells. Invasion and migration assay results revealed that knockdown of FNDC3B markedly reduced the invasive and migratory abilities of TCA8113 and TSCCA cells (Fig. 5).
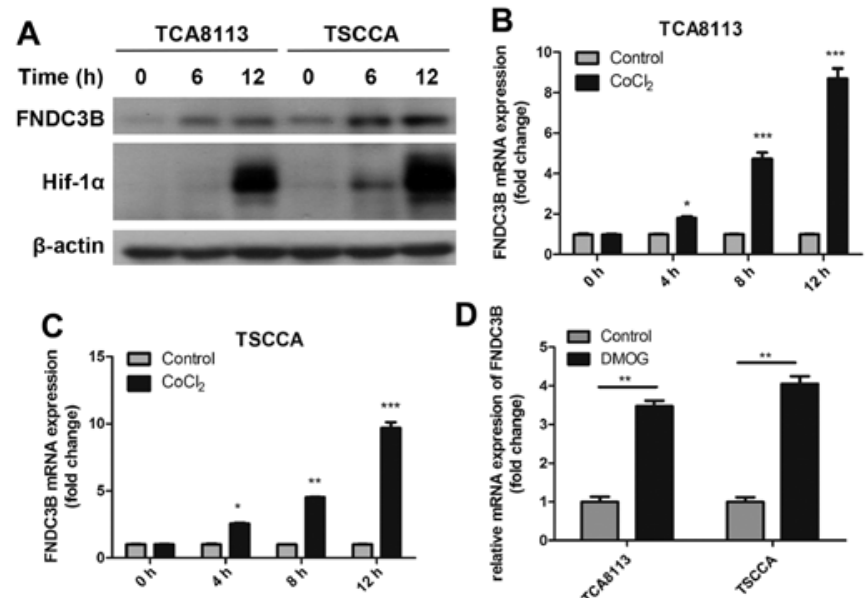

Figure 6. $\mathrm{CoCl}_{2}$ induces FNDC3B expression. (A) Protein expression and relative mRNA levels of FNDC3B in (B) TCA8113 and (C) TSCCA cells induced by $0.1 \mathrm{mM} \mathrm{CoCl}_{2}$ at the indicated times, as measured by real-time PCR and western blotting. (D) Protein expression of FNDC3B in TCA8113 cells induced by $1 \mathrm{mM}$ DMOG. ${ }^{*} \mathrm{P}<0.05,{ }^{* *} \mathrm{P}<0.01,{ }^{* * * *} \mathrm{P}<0.001$ compared to the relevant control.
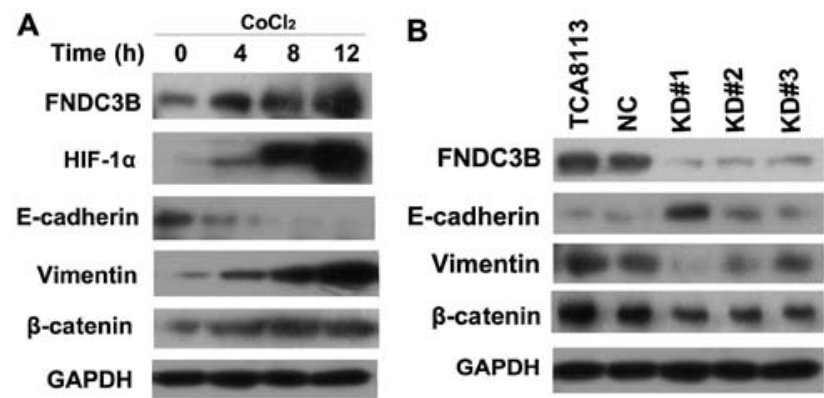

Figure 7. $\mathrm{CoCl}_{2}$ promotes EMT via inducing $\mathrm{FNDC} 3 \mathrm{~B}$ expression. (A) Western blotting of TCA8113 cells after treatment with $0.1 \mathrm{mM} \mathrm{CoCl}$ for $12 \mathrm{~h}$ using the indicated antibodies. (B) Western blotting of FNDC3B in shRNA- or NC-transfected TCA8113 cells using the indicated antibodies.

Taken together, these results suggest that FNDC3B promotes OTSCC cell invasion and migration.

Hypoxia promotes FNDC3B expression in OTSCC cells via $H I F-1 \alpha$. Hypoxia microenvironment plays an important role during the metastasis of malignancies. To identify the relationship between hypoxia and FNDC3B, we treated OTSCC cells with $0.1 \mathrm{mM} \mathrm{CoCl}_{2}$ (a hypoxia mimetic) to simulate chemical hypoxia. Treatment with $\mathrm{CoCl}_{2}$ significantly increased FNDC3B mRNA and protein production in a time-dependent manner (Fig. 6A-C). Further evidence in support of the involvement of HIF-1 $\alpha$ in the hypoxic induction of FNDC3B mRNA was provided by experiments conducted in the presence of dimethyloxallyl glycine (DMOG, $1 \mathrm{mM}$ ), which blocks degradation of HIF-1 $\alpha$ and promotes normoxic accumulation of HIF-1 $\alpha$ (Fig. 6D). Levels of FNDC3B mRNA increased by 3 - to 4 -fold in cells treated with DMOG in normal oxygen condition, relative to the untreated cells. These findings suggest that hypoxia promotes FNDC3B expression in OTSCC cells via HIF-1 $\alpha$ induction.

FNDC3B promotes EMT in a hypoxic environment. Hypoxia can promote EMT in many cancer cell types via HIF-1 $\alpha$ 
induction. Thus, we investigated the role of FNDC3B in $\mathrm{HIF}-1 \alpha$-induced EMT. We treated TCA8113 cells with $\mathrm{CoCl}_{2}$ $(0.1 \mathrm{mM})$. We found that $\mathrm{CoCl}_{2}$ upregulated HIF-1 $\alpha$ expression in OTSCC cells, meanwhile it significantly decreased E-cadherin expression and increased vimentin and $\beta$-catenin expression compared with the NC cells (Fig. 7A). On the other hand, we found that cells with FNDC3B knockdown exhibited significantly increased E-cadherin expression and decreased vimentin expression (Fig. 7B). These results demonstrated that FNDC3B plays an important role in the process of hypoxiainduced EMT in TCA8113 cells.

\section{Discussion}

Metastasis and replase are the main reasons of treatment failure for the patients with oral tongue squamous cell carcinoma (OTSCC). Lymph node metastasis is the primary factor influencing the prognosis $(1,5)$. Despite its clinical importance, little is known about the genetic and biochemical determinants of OTSCC metastasis. Thus, clarifying the mechanism of OTSCC metastasis is of significance in improving prognosis of OTSCC patients. The present study found that FNDC3B is overexpression in OTSCC tissues and positive FNDC3B expression is associated with lymph node metastasis and clinical cTNM stage.

The process of metastasis is highly complex and occurs through a series of sequential steps, including tumor cell detachment from the primary tumor; invasion through the extracellular matrix, basement membrane and endothelial wall; entry into the vascular system; secondary site plantation and growth in a target organ $(18,28)$. However, this process is also highly inefficient as few cells that migrate from the primary tumor successfully colonize at distant sites. Nearly $50 \%$ of OTSCC patients have lymph node metastases, indicating that OTSCC cells have strong invasive and migratory capacities. For cellular progression, epithelial cancer cells must undergo increased invasive and migratory abilities during EMT, which is an essential step for metastatic spreading $(19,20)$. EMT is a multi-step process where epithelial cells acquire a mesenchymal phenotype which is characterized by enhanced motility ability, downregulation of epithelial proteins including E-cadherin, claudins and $\alpha$-catenin, and overexpression of mesenchymal phenotype proteins, such as vimentin, N-cadherin and fibronectin. These changes are activated by transcription factors, including Twist1, Snail, Slug and $\beta$-catenin $(21,29)$.

Tissue hypoxia is a common microenvironment for solid tumors with rapid growth (16). The present study showed that OTSCC cells upregulate FNDC3B expression in a time-dependent manner under conditions that mimic hypoxia (treatment with $\mathrm{CoCl}_{2}$ ). During this process, mimicking hypoxia (via treatment with $\mathrm{CoCl}_{2}$ ) promotes EMT and enhances invasion and migration of OTSCC cells. These results indicate that hypoxic conditions increase FNDC3B expression, subsequently induce EMT in OTSCC cells, ultimately improving their invasive and migratory potentials.

Our data suggest the important role of FNDC3B in promoting the migratory and invasive abilities of OTSCC cells and are consistent with the findings of numerous previous studies $(12,13,30)$. FNDC3B is usually amplified and highly expressed in esophageal, lung, glioblastoma, hepatocellular and breast cancers (11-15). Lin et al (12) found that overexpression of FNDC3B facilitated cell migration and tumor metastasis in hepatocellular carcinoma. Cai et al (30) found that the $3 q$ amplified oncogene FNDC3B promoted proferiration of hepatocellular carcinoma cells, and activated several cancer pathways, including PI3-kinase/Akt, Rb1 and TGF $\beta$ signaling. MicroRNA-129-5p inhibits cell processes including viability, proliferation, migration and invasiveness of glioblastoma cells U87 through targeting FNDC3B (13). In addition, direct evidence from our in vitro experiments indicates that FNDC3B promotes cell migration and tumor metastasis via activation of EMT in OTSCC.

In conclusion, to the best of our knowledge, this is the first study to show that FNDC3B expression is positively correlated with the rate of lymph node metastasis and CTNM stage in patients with OTSCC. The mechanism underlying the FNDC3B-mediated promotion of metastasis may be related to hypoxia-induced stimulation of FNDC3B production, which promotes OTSCC cell migration, invasion and EMT. This study demonstrated that FNDC3B is a potential therapeutic target for cancer cell metastasis. However, many related issues, such as the exact mechanism of the hypoxia-induced overexpression of FNDC3B and other signaling pathways that may be activated in the process of FNCD3B-induced EMT, warrant further research.

\section{Acknowledgements}

The present study was supported by National Natural Science Foundation of China (81560470 and 81260402).

\section{References}

1. Torre LA, Bray F, Siegel RL, Ferlay J, Lortet-Tieulent J and Jemal A: Global cancer statistics, 2012. CA Cancer J Clin 65: 87-108, 2015.

2. Otsuka Y, Sato H, Oikawa T, Onodera Y, Nam JM, Hashimoto A, Fukunaga K, Hatanaka KC, Hatanaka Y, Matsuno Y, et al: High expression of EPB41L5, an integral component of the Arf6driven mesenchymal program, correlates with poor prognosis of squamous cell carcinoma of the tongue. Cell Commun Signal 14: 28, 2016.

3. Burusapat C, Jarungroongruangchai $\mathrm{W}$ and Charoenpitakchai M: Prognostic factors of cervical node status in head and neck squamous cell carcinoma. World J Surg Oncol 13: 51, 2015.

4. Haksever M, Inançlı HM, Tunçel U, Kürkçüoğlu SS, Uyar M, Genç $\mathrm{O}$ and Irkkan $\mathrm{C}$ : The effects of tumor size, degree of differentiation, and depth of invasion on the risk of neck node metastasis in squamous cell carcinoma of the oral cavity. Ear Nose Throat J 91: 130-135, 2012

5. Feng HJ, Bao YL, Liang ZP, Zhao FP, Xu SE, Xu W, Zhao C and Qin G: Silencing of FANCD2 enhances the radiosensitivity of metastatic cervical lymph node-derived head and neck squamous cell carcinoma HSC-4 cells. Int J Oncol 50: 1241-1250, 2017.

6. Katoh D, Nishizuka M, Osada S, Imagawa M and Imagawa M: Fad104, a positive regulator of adipocyte differentiation, suppresses invasion and metastasis of melanoma cells by inhibition of STAT3 activity. PLoS One 10: e0117197, 2015.

7. Wang HY, McMahon C, Ali SM, Young LE, Yekezare S, Ross JS and Ball ED: Novel FNDC3B and MECOM fusion and WT1 L378fs* 7 frameshift mutation in an acute myeloid leukaemia patient with cytomorphological and immunophenotypic features reminiscent of acute promyelocytic leukaemia. Br J Haematol 172: 987-990, 2016.

8. Nishizuka M, Kishimoto K, Kato A, Ikawa M, Okabe M, Sato R, Niida H, Nakanishi M, Osada S and Imagawa M: Disruption of the novel gene fad104 causes rapid postnatal death and attenuation of cell proliferation, adhesion, spreading and migration. Exp Cell Res 315: 809-819, 2009. 
9. Kishimoto K, Nishizuka M, Ueda T, Kajita K, Ugawa S, Shimada S, Osada S and Imagawa M: Indispensable role of factor for adipocyte differentiation 104 (fad104) in lung maturation. Exp Cell Res 317: 2110-2123, 2011.

10. Urtreger AJWS, Werbajh SE, Verrecchia F, Mauviel A, Puricelli LI, Kornblihtt AR and Bal de Kier Joffé ED: Fibronectin is distinctly downregulated in murine mammary adenocarcinoma cells with high metastatic potential. Oncol Rep 16: 1403-1410, 2006

11. Lin CH, Lin YW, Chen YC, Liao CC, Jou YS, Hsu MT and Chen CF: FNDC3B promotes cell migration and tumor metastasis in hepatocellular carcinoma. Oncotarget 7: 49498-49508, 2016.

12. Yang Y, Li D, Yang Y and Jiang G: An integrated analysis of the effects of microRNA and mRNA on esophageal squamous cell carcinoma. Mol Med Rep 12: 945-952, 2015.

13. $\mathrm{Xu} \mathrm{H}, \mathrm{Hu}$ Y and Qiu W: Potential mechanisms of microRNA$129-5 \mathrm{p}$ in inhibiting cell processes including viability, proliferation, migration and invasiveness of glioblastoma cells U87 through targeting FNDC3B. Biomed Pharmacother 87: 405-411, 2017.

14. Stangeland B, Mughal AA, Grieg Z, Sandberg CJ, Joel M, Nygård S, Meling T, Murrell W, Vik Mo EO and Langmoen IA: Combined expressional analysis, bioinformatics and targeted proteomics identify new potential therapeutic targets in glioblastoma stem cells. Oncotarget 6: 26192-26215, 2015.

15. Sawey ET, Chanrion M, Cai C, Wu G, Zhang J, Zender L, Zhao A, Busuttil RW, Yee H, Stein L, et al: Identification of a therapeutic strategy targeting amplified FGF19 in liver cancer by Oncogenomic screening. Cancer Cell 19: 347-358, 2011.

16. Ibrahim AA, Schmithals C, Kowarz E, Köberle V, Kakoschky B, Pleli T, Kollmar O, Nitsch S, Waidmann O, Finkelmeier F, et al: Hypoxia causes down-regulation of Dicer in hepatocellular carcinoma, which is required for up-regulation of hypoxia inducible factor 1alpha and epithelial-mesenchymal transition. Clin Cancer Res 23: 3896-3905, 2017.

17. Ye LY, Chen W, Bai XL, Xu XY, Zhang Q, Xia XF, Sun X, Li GG, $\mathrm{Hu} \mathrm{QD}, \mathrm{Fu} \mathrm{QH}$, et al: Hypoxia-induced epithelial-to-mesenchymal transition in hepatocellular carcinoma induces an immunosuppressive tumor microenvironment to promote metastasis. Cancer Res 76: 818-830, 2016

18. Zhong Z, Hu Z, Jiang Y, Sun R, Chen X, Chu H, Zeng M and Sun C: Interleukin-11 promotes epithelial-mesenchymal transition in anaplastic thyroid carcinoma cells through PI3K/Akt/ GSK3 $\beta$ signaling pathway activation. Oncotarget 7: 59652-59663, 2016.

19. Wu Y, Wang Y, Lin Y, Liu Y, Wang Y, Jia J, Singh P, Chi YI, Wang C, Dong C, et al: Dub3 inhibition suppresses breast cancer invasion and metastasis by promoting Snaill degradation. Nat Commun 8: 14228, 2017. doi: 10.1038/ncomms14228.
20. Zhang T, Liang L, Liu X, Wu JN, Chen J, Su K, Zheng Q, Huang H and Liao GQ: TGF 31 -Smad3-Jagged1-Notch1-Slug signaling pathway takes part in tumorigenesis and progress of tongue squamous cell carcinoma. J Oral Pathol Med 45: 486-493, 2016.

21. Shi ZM, Wang L, Shen H, Jiang CF, Ge X, Li DM, Wen YY, Sun HR, Pan MH, Li W, et al: Downregulation of miR-218 contributes to epithelial-mesenchymal transition and tumor metastasis in lung cancer by targeting Slug/ZEB2 signaling. Oncogene 36: 2577-2588, 2017.

22. Edge SB, Byrd DR, Compton CC, Fritz AG, Greene FL and Trotti A: AJCC Cancer Staging Manual. 7th edition. Springer, New York, 2010.

23. Estilo CL, O-charoenrat P, Talbot S, Socci ND, Carlson DL, Ghossein R, Williams T, Yonekawa Y, Ramanathan Y, Boyle JO, et al: Oral tongue cancer gene expression profiling: Identification of novel potential prognosticators by oligonucleotide microarray analysis. BMC Cancer 9: 11, 2009.

24. Kuriakose MA, Chen WT, He ZM, Sikora AG, Zhang P, Zhang ZY, Qiu WL, Hsu DF, McMunn-Coffran C, Brown SM, et al: Selection and validation of differentially expressed genes in head and neck cancer. Cell Mol Life Sci 61: 1372-1383, 2004.

25. Pyeon D, Newton MA, Lambert PF, den Boon JA, Sengupta S, Marsit CJ, Woodworth CD, Connor JP, Haugen TH, Smith EM, et al: Fundamental differences in cell cycle deregulation in human papillomavirus-positive and human papillomavirus-negative head/neck and cervical cancers. Cancer Res 67: 4605-4619, 2007.

26. Talbot SG, Estilo C, Maghami E, Sarkaria IS, Pham DK, O-charoenrat P, Socci ND, Ngai I, Carlson D, Ghossein R, et al: Gene expression profiling allows distinction between primary and metastatic squamous cell carcinomas in the lung. Cancer Res 65: 3063-3071, 2005.

27. Ye H, Yu T, Temam S, Ziober BL, Wang J, Schwartz JL, Mao L, Wong DT and Zhou X: Transcriptomic dissection of tongue squamous cell carcinoma. BMC Genomics 9: 69, 2008.

28. Książkiewicz M, Markiewicz A and Zaczek AJ: Epithelialmesenchymal transition: A hallmark in metastasis formation linking circulating tumor cells and cancer stem cells. Pathobiology 79: 195-208, 2012.

29. Nieto MA: Context-specific roles of EMT programmes in cancer cell dissemination. Nat Cell Biol 19: 416-418, 2017.

30. Cai C, Rajaram M, Zhou X, Liu Q, Marchica J, Li J and Powers RS: Activation of multiple cancer pathways and tumor maintenance function of the $3 \mathrm{q}$ amplified oncogene FNDC3B. Cell Cycle 11: 1773-1781, 2012. 